\title{
Interfaces entre a Avaliação Psicológica e a Clínica Psicanalítica
}

\section{Resumo}

\author{
Helena Rinaldi Rosa* \\ Leila Salomão De La Plata Cury Tardivo** \\ Antonio Augusto Pinto Junior*** \\ Marlene Alves da Silva**** \\ Hilda Rosa Capelão Avoglia *****
}

Este trabalho visa refletir sobre as interfaces entre a clínica psicanalítica e a avaliação psicológica, mostrando a possibilidade de diálogo e de encontro entre ambas e que dados de pesquisas científicas podem contribuir com a compreensão clínica de pessoas com dificuldades emocionais. Método: Trata-se de um estudo teórico com esse objetivo e, para isso os Resultados e Discussão ocorrem por meio da apresentação de algumas pesquisas científicas quantitativas, realizadas pela autora em meio acadêmico, com o teste do Desenho da Figura Humana (DFH)e um breve estudo de caso no qual foi feita a avaliação psicológica com diversos instrumentos, incluindo o DFH. Os diferentes trabalhos apresentados são discutidos em função de como indicam as interfaces da avaliação psicológica com a clínica psicanalítica, e de como a primeira subsidia a compreensão e a ação na atuação psicoterapêutica infantil, revendo as ideias prévias, permitindo à criança e sua família a possibilidade de pensar.

Palavras-chave: Psicanálise. Avaliação Psicológica. Desenho De Figuras Humanas.

\section{Interfaces Between Psychological Assessment and the Psychoanalytic Clinic}

\begin{abstract}
This article aims to reflect on the interfaces between the psychoanalytic clinic and the psychological assessment, showing the possibility of dialogue and encounter between both, and also demonstrate that data from scientific research can also contribute to the clinical understanding of people with emotional difficulties. This is done through the presentation of some quantitative scientific research carried out by the author in an academic environment, using the Human Figure Drawing Test (DFH), and the brief presentation of a case study in which this test was used. Results and Discussion: The different researches presented are discussed in terms of how the interfaces between psychological assessment and psychoanalytic clinic indicate, and how one supports understanding and action in child psychotherapeutic activity, allowing the child and the Family to think.
\end{abstract}

Keywords: Psychoanalysis. Psychological Evaluation. Drawing of Human Figures.

* Psicóloga, Mestre, Doutora e Professora do Instituto de Psicologia da USP.

** Psicóloga, Mestre, Doutora, Livre Docente e Professora do Instituto de Psicologia da USP.

*** Psicólogo pela Faculdade Salesiana de Filosofia Ciências e Letras de Lorena-SP. Mestre e Doutor pela USP. Docente associado II da UFF.

**** Psicóloga pela Universidade Metodista de São Paulo. Mestre e Doutora pela Universidade São Francisco de Itatiba - SP. Pós Doutoranda na USP.

****** Psicóloga e Mestre pela Universidade Metodista de São Paulo. Doutora em Psicologia pela USP. Docente dos Programas de Mestrado em Psicologia da Saúde - UMESP e Psicologia e Políticas Públicas da Universidade Católica de Santos. 
O objetivo deste trabalho é promover uma aproximação entre a psicanálise e a pesquisa acadêmica e demonstrar as possibilidades de encontro criativo entre ambas. O lugar de onde se fala é o de docentes e pesquisadores na área da avaliação psicológica, área de atuação dos pesquisadores há muitos anos. Pretende-se focalizar a clínica com crianças por ser o público com o qual mais se trabalha, mas as considerações feitas aqui se aplicam igualmente aos adultos. Em ambos os casos, as queixas e dificuldades estão sempre no limiar entre o mundo externo e o mundo interno. É precisamente essa área intermediária que a avaliação psicológica pretende explorar.

Quando o profissional recebe uma criança, trazida pelos pais ou responsáveis, é comum que ela chegue já diagnosticada com algum tipo de transtorno: aprendizagem, atenção, concentração ou outras nomenclaturas que vão surgindo, como transtornos de oposição ou desafiante. Tais rótulos costumam ser frutos de conclusões de outro profissional, e também por leigos na intenção de ajudar, tais como professores e representantes das escolas, quando não pelos próprios pais. Assim, é fundamental que se questione esses diagnósticos e se vá em busca do que de fato ocorreu com a criança. A expressão "de fato" é necessária e indispensável quando se pensa no que pode estar ocorrendo (hipóteses) e como prestar um serviço à criança e sua família.

Os instrumentos de avaliação psicológica podem ser um recurso para o questionamento objetivo desses diagnósticos, fornecendo, assim, suporte à avaliação clínica. Contudo, historicamente, os testes psicológicos foram muito criticados por seu viés positivista e por não considerarem os aspectos subjetivos presentes no contexto investigado (Silva, 2016). Quando a Psicologia estava ainda se estabelecendo como ciência e como profissão, no século passado, foi por meio dos testes psicológicos, fundamentados em modelos de outras ciências, que ela aparentemente conquistou seu lugar. A hipervalorização dessas técnicas, num primeiro momento, foi sucedida por questionamentos ainda mais intensos - e procedentes, sobre a sua própria eficácia. Os testes prometiam muito mais do que faziam. $\mathrm{Na}$ atualidade, a avaliação psicológica volta a tomar força, procurando se sustentar em bases científicas mais sólidas e incluindo sistematicamente os fatores subjetivos, intersubjetivos e o contexto vivido pela pessoa a ser avaliada.

\section{Objetivo}

Dessa forma, o objetivo deste trabalho foi, ao apresentar de forma breve diferentes pesquisas em Avaliação
Psicológica, discutir o entrelaçamento desta área com a clínica psicanalítica, demonstrando que uma avaliação objetiva bem cuidada e rigorosa pode contribuir na compreensão dos aspectos intersubjetivos presentes nos casos clínicos.

\section{Método}

Quanto aos aspectos metodológicos, este trabalho apresenta algumas pesquisas feitas com o Teste do Desenho da Figura Humana (DFH), com a finalidade de discutir suas possibilidades de uso na clínica psicanalítica, ilustrando com um caso clínico; portanto, é um artigo teórico - ainda que comentando as pesquisas de campo anteriormente apresentadas.

As pesquisas abordadas nesse trabalho têm se concentrado no DFH, de destaque entre os testes psicológicos por seu baixo custo, relativa facilidade de interpretação e, sobretudo, por ser bem aceito pelas crianças, sendo um dos desenhos que elas mais fazem tanto na escola como em contexto clínico. Existe uma longa história de estudos realizados, inicialmente avaliando o desenhista do ponto de vista cognitivo e desenvolvimental como Goodenough (1926); Harris (1963); Koppitz (1968); Naglieri (1988) e, ainda, no Brasil, Wechsler (2003); Sisto (2005) e Rosa (2018).

São muitos os estudos que investigam como as crianças desenham, evoluindo de um traço que é praticamente uma descarga motora (no bebê) até o desenho completo de uma pessoa, incluindo aspectos como perfil, roupas, presença de duas dimensões, o que ocorre por volta dos 11 ou 12 anos de idade (Koppitz, 1968 e Rosa, 2018). A partir dessa idade, a estabilidade no desenho se instala e o DFH é usado, com muito maior eficácia, para avaliação de aspectos emocionais. A ideia é de que há um paralelismo entre o desenvolvimento cognitivo e a evolução do grafismo.

Foram empregados dois sistemas diferentes de avaliação cognitiva do Desenho de um Homem e um teste não verbal de inteligência (Teste R-2), com uma amostra de 1.540 crianças, de 5 a 11,5 anos, de ambos os sexos, sorteadas de modo a ser representativa de escolares da cidade de São Paulo (Rosa, 2006). Foram controladas as variáveis: idade, sexo e tipo de escola frequentada pela criança, este último dado tomado como indicador do nível socioeconômico.

As médias de pontos mostraram crescimento progressivo com a idade pelos dois sistemas de avaliação, como também ocorre com testes de inteligência, em que a pontuação aumenta com a idade. Foram estabelecidas 
normas para os dois sistemas de avaliação por idade e separadas por sexo nas idades em que houve diferenças significantes entre os sexos. O tipo de escola não apresentou diferenças com significância estatística, sugerindo que o desenho pode estar associado em maior grau a fatores maturacionais do que a ambientais.

\section{Investigação e avaliação dos fatores emocionais no DFH}

A partir dessa pesquisa, têm sido realizados diferentes estudos no Laboratório Interdepartamental de Técnicas de Exame Psicológico, entre eles: (1) o sistema de avaliação cognitiva de Koppitz (1968) e sua relação com o sistema atualmente aprovado para uso clínico segundo o Conselho Federal de Psicologia (DFH-III, proposto por Wechsler em 2003); e (2) o estudo dos Indicadores Emocionais do DFH desenvolvido por Koppitz (1968), uma vez que normas para tais indicadores junto à população da cidade de São Paulo foram estabelecidas por Kobayashi (2015), porém sem investigar amostras clínicas. A última autora estabeleceu, para a população infantil paulistana, as idades em que os IE podem ser considerados Indicadores Emocionais, em razão de serem ou não esperados para aquela idade.

Assim, estudos de casos clínicos também têm sido realizados e apresentados em eventos científicos, bem como comparativos entre variadas amostras, inclusive de regiões diferentes do país, de crianças matriculadas em escolas públicas e particulares, amostras clínicas comparadas com não clínicas, entre outras variáveis. Trata-se de uma área que demanda investigação, uma vez que não há na literatura concordância sobre os Indicadores Emocionais, como a existente sobre os Indicadores Maturacionais (Rosa, 2018).

São apresentados, a seguir, suscintamente, os aspectos metodológicos envolvidos nesse tipo de pesquisa, alguns dos resultados que vêm sendo obtidos, num modelo quantitativo e, na sequência, em um estudo de caso. No sistema de pontuação empregado na avaliação psicológica infantil do Desenho da Figura Humana (DFH) proposto por Koppitz (1968) - um dos mais utilizados internacionalmente, são verificados os Indicadores Maturacionais, a fim de avaliar o desenvolvimento da criança, e os Indicadores Emocionais, para determinar comprometimentos nessa área. São atribuídos pontos para a presença de cada item no desenho e o total de pontos é analisado segundo a tabela normativa para a idade e sexo do sujeito. O mesmo procedimento foi empreendido na proposta de Wechsler (2003), brasileira, aprovada pelo Conselho de Psicologia, o DFH-III.
Enquanto Koppitz (1968) solicita apenas um desenho da Figura Humana, para determinar a presença de Indicadores Maturacionais e de Indicadores Emocionais, com 30 itens para cada uma dessas escalas, o DFH-III (Wechsler, 2003) solicita dois desenhos, um do homem e um da mulher e pontua 58 itens para cada figura, que avaliam a maturidade. Por essa razão, solicitou-se os dois desenhos e não apenas o desenho de uma pessoa, como propôs Koppitz, com o intuito de comparar os desenhos do homem e da mulher. A Tabela 1 apresenta alguns dos Indicadores Maturacionais de Koppitz (1968) e do DFH-III, a fim de ilustrar o que está sendo pontuado em cada sistema.

\section{Tabela 1 - Exemplos de Indicadores Maturacionais de Koppitz (1968) e seus correspondentes no DFH-III}

\begin{tabular}{|c|c|}
\hline KOPPITZ & DFH-III \\
\hline 1. Cabeça & $\begin{array}{l}\text { 1. Cabeça: } \\
\text { a) ( ) presença } \\
\text { b) ( ) proporção [sendo na figura feminina, se a cabeça } \\
\begin{aligned} \text { tem a forma ovalada e mais comprida do que } \\
\text { larga (item } 8 \text { a na masculina) - e } 1 \text { b é se a cabeça } \\
\\
\text { tem } 1 / 3 \text { do corpo] }\end{aligned}\end{array}$ \\
\hline 10. Cabelo & $\begin{array}{l}\text { 2. Cabelo: } \\
\begin{array}{l}\text { a) ( ) presença } \\
\text { b) ( ) adaptação } \\
\text { c) ( ) penteado }\end{array}\end{array}$ \\
\hline 2. Olhos & $\begin{array}{l}\text { 3. Olhos: } \\
\text { a) ( ) presença } \\
\text { b) ( ) sobrancelhas } \\
\text { c) ( ) pupilas } \\
\text { d) ( ) proporção } \\
\text { e) ( ) direção }\end{array}$ \\
\hline 4. Nariz & $\begin{array}{l}\text { 4. Nariz: } \\
\begin{array}{l}\text { a) ( ) presença } \\
\text { b) ( ) duas dimensões } \\
\text { c) ( ) estrutura completa } \\
\text { d) ( ) fossas nasais }\end{array}\end{array}$ \\
\hline
\end{tabular}

Os itens apresentados na Tabela 1 são apenas alguns entre os 30 itens propostos por Koppitz (1968) e os 58 itens no DFH-III, com a correspondência entre eles. Pode-se observar que o DFH-III apresenta itens muito semelhantes ao primeiro, porém com maior detalhamento. O total de pontos é comparado com tabelas normativas para a população e pode-se, assim, inferir se o desenho 
de uma criança está dentro do esperado para a sua idade (média para aquela idade), acima ou abaixo. Cabe ressaltar que os itens não estão todos apresentados porque os testes psicológicos são privativos do psicólogo e é proibida a sua veiculação a profissionais não psicólogos, que é o público-alvo desta publicação.

Enquanto se espera maior número de Indicadores Maturacionais com o crescimento, espera-se menor número de Indicadores Emocionais, não sendo desejável que apareçam no desenho, na medida em que sugerem dificuldades. A escala de Indicadores Emocionais proposta por Koppitz apresenta como características: serem raros, isto é, apresentarem frequência menor do que 16\% na população geral; diferenciarem as crianças com e sem problemas emocionais; e não apresentarem aumento da frequência com a progressão da idade. A Tabela 2 mostra alguns dos IEs.

Tabela 2 - Indicadores Emocionais de Koppitz (1968)

\begin{tabular}{c|l}
\hline Itens & Indicadores \\
1 a 4 & Sombreamentos \\
5 & Assimetria grosseira das extremidades \\
6 & Figura inclinada em $15^{\circ}$ ou mais \\
7 & Figura pequena, com $5 \mathrm{~cm}$ ou menos \\
8 & Figura grande, com $23 \mathrm{~cm}$ ou mais \\
18 & Pernas apertadas \\
19 & Genitais \\
20 & Monstro ou figura grotesca \\
21 & Desenho espontâneo de três ou mais figuras \\
22 & Nuvens, chuva e neve \\
23 a 30 & Omissões \\
\hline
\end{tabular}

Os itens propostos por Koppitz (1968) para avaliação de dificuldades emocionais foram investigados em pesquisa do Laboratório Interdepartamental de Técnicas de Exame Psicológico (LITEP), comparando uma amostra de 74 crianças, na faixa etária de 6 a 11 anos, que buscaram atendimento psicológico (consultórios particulares e clínicas-escolas) com queixas diversas, com um grupo controle de 74 escolares emparelhados por idade, sexo e tipo de escola, sem queixas emocionais. A aplicação foi individual e ocorreu nas clínicas de atendimento para o primeiro grupo e nas escolas para o grupo controle, garantindo que não houvesse prejuízos às atividades escolares.

O projeto foi aprovado pelo Comitê de Ética em Pesquisa com Seres Humanos. As crianças do primeiro grupo apresentavam diversas queixas psicológicas (dificuldades escolares, medos e ansiedade, queixas somáticas, problemas de comportamento, agitação, timidez e retraimento, entre outras). As do segundo, não apresentavam queixas de dificuldades escolares ou de comportamento, segundo os professores e a direção das escolas.

\section{Resultados e Discussão}

Tanto no desenho do homem quanto no da mulher, as crianças do grupo clínico tiveram um total de pontos, em média, menor que o grupo de crianças sem queixas emocionais (grupo controle), e essa diferença foi significante estatisticamente (no homem $p=0,005$, na mulher, $\mathrm{p}<0,001)$. Esse resultado sugere que as crianças do grupo clínico têm mais prejuízos no desenvolvimento cognitivo, o que se relaciona, possivelmente, às dificuldades emocionais.

Também ocorreram diferenças significantes entre as médias de pontos de Indicadores Emocionais de ambos os grupos, para as duas figuras. Koppitz (1968) constatou que, para diagnosticar a presença de problemas emocionais, era necessário um mínimo de dois indicadores. As crianças da amostra clínica obtiveram média maior de indicadores (1,82 para o homem e 2,01 para a mulher) em comparação aos sem queixas e só atingiram os dois pontos estabelecidos por Koppitz no desenho da mulher, ao passo que os escolares obtiveram em média 1,2 IE para cada uma das duas figuras. Pode-se concluir que tais resultados corroboram a afirmação de Koppitz de que os Indicadores Emocionais são sensíveis para diferenciar as duas amostras, constituindo evidência da validade dos Indicadores Emocionais de Koppitz para o diagnóstico de problemas emocionais em crianças, ao menos na faixa etária estudada.

As correlações entre os Indicadores Maturacionais e Emocionais para cada desenho em cada grupo foram muito próximas, mostrando a existência de uma relação entre os dois tipos de indicadores. Esse resultado aponta que os IMs são afetados pelos IEs, o que está de acordo com os resultados de Wechsler, Prado, Oliveira e Mazzarino (2011), cuja pontuação emocional também se correlacionou negativamente com a cognitiva, sugerindo que as dificuldades emocionais (pontos atribuídos à presença de Indicadores Emocionais) podem trazer prejuízos no desempenho das crianças ou, ainda, dificuldades cognitivas podem levar a comprometimentos emocionais.

A fim de investigar a influência do nível socioeconômico nos resultados do teste, estes foram investigados quanto ao tipo de escola que frequentam as crianças, que não têm queixas de problemas emocionais. Assim, pretendeu-se investigar a sensibilidade das propostas do 
DFH-III (Wechsler, 2003) e de Koppitz (1968) para identificar diferenças socioeconômicas. Foram comparados os desenhos de 100 escolares, de 6 a 10 anos de idade, de ambos os sexos igualmente, metade de escolas públicas e metade particulares. As correlações entre os dois sistemas foram altas e significantes $(p<0,001)$, tanto para o desenho do homem quanto para o da mulher. Houve crescimento das médias com o aumento da idade, confirmando o teste como instrumento de avaliação desenvolvimental.

Nos Indicadores Maturacionais de Koppitz, as médias de pontos foram ligeiramente maiores para as crianças de escolas particulares, porém a diferença entre os tipos de escola não foi estatisticamente significante, sugerindo que, ao menos com a amostra empregada, a variável nível socioeconômico não exerce influência no desempenho das crianças no desenho avaliado segundo Koppitz. Houve uma diferença significante entre os sexos apenas para o desenho da mulher, os outros testes não mostraram diferenças significantes entre as crianças do sexo masculino e feminino. Entretanto, houve diferenças entre os tipos de escola quando os mesmos desenhos foram avaliados pelo DFH-III. Os resultados sugerem uma sensibilidade superior do sistema de pontuação DFH-III na identificação de diferenças socioeconômicas em relação ao sistema de Koppitz.

Foram apresentados dois recortes de pesquisas com o DFH cujos resultados podem ajudar o clínico a confrontar aquele que avalia com o que se observa nas pesquisas acadêmico científicas. Os pesquisadores vêm se debruçando sobre diferenças regionais, investigando-as quanto ao contexto cultural, comparando amostras de crianças da cidade de São Paulo com as de outra cidade, no caso, a de Aracaju (SE), e os resultados preliminares indicam, nos dois sistemas, prejuízos para a população do Nordeste - controlando a idade, o sexo e o tipo de escola que as crianças frequentam. Pretende-se, ainda, avaliar amostras clínicas com queixas específicas para comparar com amostra controle, como é o caso de crianças com TDAH, entre outros casos.

Cabe mencionar que resultados pontuais em testes psicológicos, até mesmo indicadores como os apresentados, não devem ser interpretados isoladamente, mas sempre em conjunto. Não apenas junto a demais testes, para se obter uma compreensão global da pessoa avaliada. A literatura mostra que os dados mais confiáveis na análise de desenhos referem-se ao aspecto geral do desenho, à sua qualidade enquanto produção do sujeito (Hammer, 1981; Cunha, 2000). Por esta razão, indicadores devem ser interpretados com base na produção como um todo.

\section{Vinheta}

A seguir, é apresentada outra pesquisa com o objetivo de investigar a validade dos itens propostos por Koppitz para avaliação desenvolvimental e de dificuldades emocionais, por meio de um estudo de caso clínico em que o DFH foi aplicado juntamente com uma bateria usual de testes em psicodiagnósticos. Foram, também, aplicados os seguintes testes: WISC-IV, Teste de Bender, Teste de Atenção, Teste das Pirâmides Coloridas de Pfister e Desenho da Família. Trata-se de uma criança do sexo masculino, com 10 anos de idade (10 anos e 7 meses por ocasião do psicodiagnóstico), com queixa de dificuldades de aprendizagem. Foram realizadas entrevistas de anamnese com pai e mãe, entrevistas com a criança e com a coordenação da escola, bem como orientações mensais aos pais para mudança de postura, diante do relato de falta de rotina e de imposição de limites. Diante das dúvidas sobre o potencial da criança, foi realizado o psicodiagnóstico, em contexto clínico segundo o modelo compreensivo e interventivo.

O menino é filho único de pais que se separaram quando ele tinha quatro anos, permanecendo com a mãe na cidade natal, distante cerca de 400 quilômetros da capital, para onde o pai mudou-se. Ele se casou novamente, e vem a cada quinzena visitar o filho, ficando com ele cerca de cinco dias. A queixa de dificuldades de aprendizagem foi apontada pela escola, entretanto a mãe não concordou, culpabilizando a escola inicialmente e depois, considerando um déficit de atenção da criança, buscando uma medicação que resolvesse tal queixa. A coordenação da escola, por sua vez, colocou que ele não participava das aulas e ficava todo o tempo conversando com os colegas. Os relatos foram de dificuldade em aceitar os limites, bem como falta de rotina (hábitos de sono, alimentação e estudo) para a criança.

$\mathrm{Na}$ observação do seu comportamento junto à psicóloga que o atendeu, mostrou-se uma criança muito infantilizada para a idade; não respeitava ou seguia regras; realizava a todo o momento brincadeiras incoerentes e "sem graça": contava mentiras e dava ênfase nos detalhes de suas histórias para que estas se tornassem mais interessantes, desta forma pretendia obter mais atenção dos demais, mas na verdade provocava tédio e afastamento. Não tinha rotina, sendo que nunca sabia onde passaria o dia e dormiria, a cada dia dormia num local diferente, em casa, na avó materna, avó paterna, na tia, etc.; diante disso não havia uma estruturação de horários a serem seguidos. Como também não sabia o que era esperado dele e se "acomodava". Acomodava-se no caos de um 
ambiente em que existiam poucas regras, sentia-se negligenciado, sem referências, e queria ser o centro das atenções dos pais e da escola.

Os resultados da avaliação feita com os instrumentos acima mencionados revelaram inteligência acima da média para crianças de sua idade, bem como sua coordenação visomotora e atenção. No teste das Pirâmides Coloridas de Pfister apresentou certa dificuldade em lidar com as emoções, mesmo em se tratando de uma criança, que buscaria assim controlar e reprimir os impulsos - não entrando em contato com os sentimentos. No desenho da família, fez uma família com pai, mãe e uma filha no meio dos pais, todos muito próximos no lado esquerdo da folha, indicando seu pedido de ajuda para lidar com o ambiente pouco estruturado em que vive.

No Teste do Desenho da Figura Humana ele obteve os seguintes resultados:

- No desenho do homem, houve a presença de um Indicador Emocional (omissão de nariz, o que não é mais esperado para sua idade). No da mulher, houve a presença de dois Indicadores Emocionais (omissão de nariz e presença de dentes) - o que, segundo a autora estudada, já é relevante para sugerir dificuldades emocionais que requerem investigação mais detalhada.

- $\mathrm{Na}$ avaliação maturacional, seu desenho do homem classificou-se como Média Inferior segundo a tabela brasileira para Koppitz (Rosa, 2006) bem como pelo DFH-III. O mesmo ocorreu no da mulher, segundo Koppitz (Rosa, 2006) porém, pelo DFH-III, a criança está dentro da média esperada para sua idade.

Figura 1

\section{Desenho de um homem}

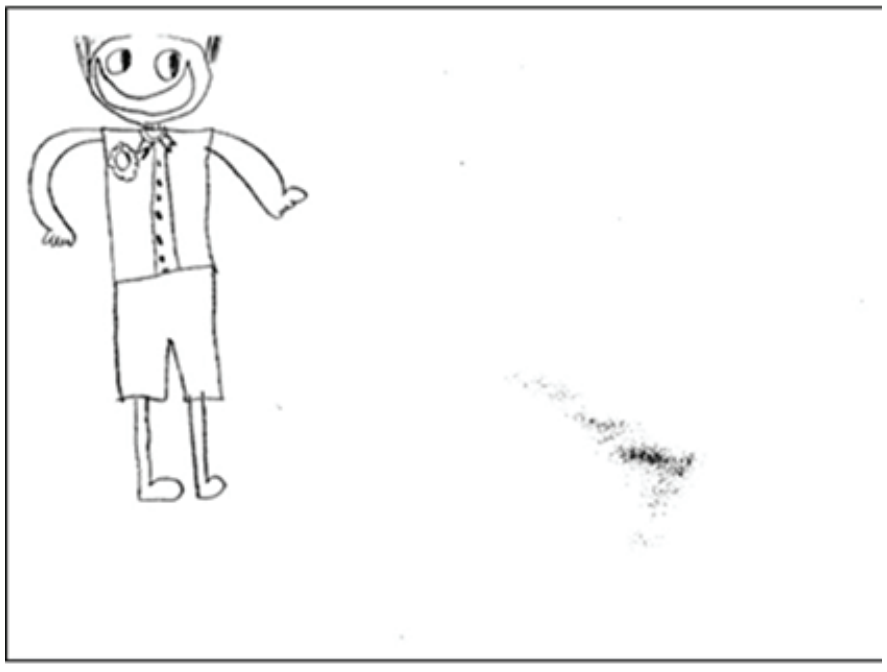

Figura 2

Desenho de uma mulher

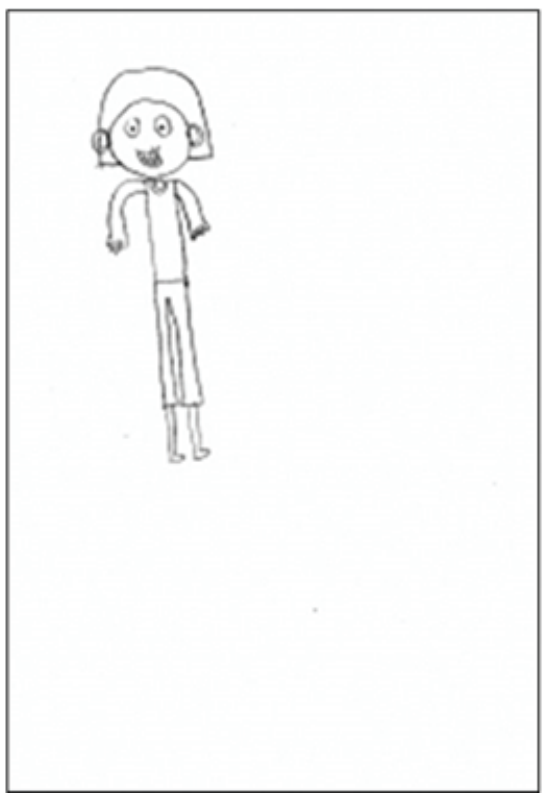

Observa-se que os resultados nos instrumentos utilizados vão ao encontro das observações da psicóloga que o atendeu. Não há indicação de problemas de transtorno de déficit de atenção, porém sugere-se a presença de questões emocionais, que se revelam em sua dificuldade em aceitar regras e também no comportamento de chamar a atenção da psicóloga e dos pais. Dificuldades cognitivas, déficit de atenção e problemas neurológicos, assim como de coordenação visomotora, são hipóteses descartadas. Isso confere maior flexibilidade para quem lida com ele, por saber que tem recursos, pode desenvolver-se e buscar melhor adaptação. $\mathrm{O}$ aspecto geral dos desenhos também mostra seu potencial e bom prognóstico.

O DFH foi importante por esclarecer ainda mais as dificuldades emocionais. Sendo o desenho mais suscetível aos problemas relacionais e afetivos, entende-se seu resultado, do ponto de vista cognitivo, inferior aos apresentados no teste de inteligência (WISC-IV). Considerando a história de vida da criança, essas dificuldades parecem ter origem numa relação com uma mãe e um pai permissivos, provavelmente com as mesmas dificuldades de lidar com limites, e possivelmente, por esse mesmo motivo, de ensiná-los ao filho.

Após perceberem o real potencial da criança, o menino pôde "renascer" aos olhos dos pais, havendo maior consideração pelos aspectos emocionais que estavam impedindo um contato mais organizado com a aprendizagem e com a realidade. Passaram a considerar a importância e o benefício de se propor limites ao invés de impô-los, assim 
como puderam estabelecer uma rotina que ele pudesse compreender. Um meio que realmente fosse um referencial compreensivo e apreensível para ele, um norteador para seu desenvolvimento. Certamente a situação vivida pela criança acarreta mais dificuldades no enfrentamento da realidade, e pode-se inferir que chegam a interferir em seu desempenho. Os resultados apresentados no psicodiagnóstico e em especial no desenho da figura humana indicam isso. Portanto, o DFH mostrou-se sensível no rastreamento das habilidades e das dificuldades da criança, confirmando sua validade clínica e sustentando a compreensão psicológica do caso no âmbito da criança. São necessários estudos com amostras mais amplas e mais diversificadas para se fazer maiores generalizações.

Dessa forma, pode-se considerar que os resultados nas diferentes pesquisas mostraram que os Indicadores Emocionais diferenciaram as crianças com dificuldades emocionais que, em geral, têm seus desenhos afetados por tais dificuldades, contribuindo para confirmar a validade do Teste do Desenho da Figura Humana para a avaliação emocional, além da desenvolvimental, em nosso país.

\section{Interfaces da avaliação psicológica com a clínica psicanalítica}

Entende-se que não é possível praticar psicanálise separadamente da investigação e que levar em conta os aspectos do mundo subjetivo da dupla com o paciente, é o que faz de melhor o analista. Navegar pelo mundo mental é tarefa árdua, que pode ser estimulada pelo desejo de saber, de compreender, que move a investigação. Dados objetivos, como os descritos e ilustrados na avaliação feita com a criança fornecem subsídios de suma importância para a clínica psicanalítica, seja com crianças, com adolescentes, ou com adultos, fornecendo ao psicanalista novas possibilidades de compreensão.

É necessário raciocínio clínico e abertura para aproveitar a avaliação psicológica sem ficar enrijecido pelas informações que este recurso fornece e numa postura aberta para o campo de hipóteses que podem ser levantadas. O encontro entre o clínico e seu cliente - tema deste trabalho - se dá a todo momento, mesmo que não tão claramente. O psicanalista e seu analisando, ao viverem sua experiência singular e única, não prescindem de uma avaliação do que está ocorrendo, todo o tempo.

\section{Considerações Finais}

Reitera-se a importância de pesquisas como as aqui apresentadas, pois enfatizam aspectos objetivos da realidade, mostram o que pode ser esperado na população, sugerindo o que pode ser uma preocupação real dos pais/ responsáveis quando trazem suas crianças com questões que, por sua vez, podem ou não ser procedentes. Diagnósticos que rotulam e estigmatizam as pessoas fecham o entendimento sobre o que ocorre com elas e pouco favorecem a compreensão clínica. E poder fornecer um dado objetivo sobre como a criança está, com relação ao seu próprio grupo, isto é, o que está dentro do esperado e o que não está, reautoriza a família a se aproximar da criança e do sintoma, tornando possível entendê-lo, emancipando e libertando o sujeito dos diagnósticos preestabelecidos que só o impedem de pensar.

\section{Referências}

Goodenough, F. L. (1926). Measurement of intelligence by drawings. NewYork: World Book C.

Harris, D. B. (1963). Children's drawings as measure of intellectual maturity. New York: Harcourt, Brace \& World.

Kobayashi, C. (2015). Os Indicadores Emocionais de Koppitz no Desenho da Figura Humana de crianças de São Paulo. Tese de Doutorado, Instituto de Psicologia, Universidade de São Paulo, São Paulo. Disponível parcialmente em https://www.teses.usp.br/teses/disponiveis/47/.../kobayashi_parcial.pdf

Koppitz, E. M. (1968). Psychological evaluation of children's Human Figure Drawings. New York: Grune \& Stratton.

Naglieri, J. (1988). Draw a Person: A quantitative scoring system. New York: Psychological Corporation.

Rosa, H. R. (2006). Teste Goodenough-Harris e Indicadores Maturacionais de Koppitz.para o Desenho da Figura Humana: Estudo Normativo para crianças de São Paulo. Tese de Doutorado, Instituto de Psicologia, Universidade de São Paulo, São Paulo. Disponível em: https://www.teses.usp.br/teses/ disponiveis/47/47131/.../1-teseHelenaRR.pdf

Rosa, H.R. (2018). Desenho da Figura Humana em crianças: Indicadores Emocionais, evidências de Validade e Precisão. Tese (de Livre Docência). Universidade de São Paulo.

Silva, M.C.V.M. (2016). História dos Testes Psicológicos: Origens e Transforma ções. Vetor.

Sisto, F. F. (2005). Desenho da Figura Humana - Escala Sisto (DFH - Escala Sisto). Manual. São Paulo: Vetor.

Wechsler, S. M. (2003). O Desenho da Figura Humana: avaliação do desenvolvimento cognitivo de crianças brasileiras (3a. ed.). Campinas: Lamp/ PUC-Campinas.

Wechsler, S. M., Prado, C. M., Oliveira, K. S., \& Mazzarino, B. G. (2011). Desenho da Figura Humana: análise da prevalência de indicadores para avaliação emocional. Psicologia: Reflexão e Crítica, 24(3), 411-418. Recuperado em 07/agosto/2013, de http://www.scielo.br/pdf/prc/v24n3/a01v24n3.pdf

Submetido em: 20-3-2020

Aceito em: 16-7-2020 